Sección Básica / Basic

Notas científicas / Scientific notes

\title{
Detección de Oryctomorphus bimaculatus (Coleoptera: Scarabaeidae: Rutelinae) en Platanus orientalis, Talca, Chile
}

\author{
Detection of Oryctomorphus bimaculatus (Coleoptera: Scarabaeidae: Rutelinae) in Platanus orientalis, Talca, Chile \\ OSCAR VALLEJOS-BARRA ${ }^{1}$, CHRISTIAN VIDAL PAIVA², MAURICIO PONCE-DONOSO ${ }^{3}$ \\ y MARÍA MENDOZA ÁLVAREZ ${ }^{4}$
}

\begin{abstract}
${ }^{1}$ Dr. Facultad de Ciencias Forestales, Universidad de Talca, Talca, Chile, 1 poniente 1141, Talca, Chile, ovallejo@utalca.cl, https://orcid.org/0000-00020155-0835. ${ }^{2}$ Ing. Forestal, consultor independiente, Talca, Chile, chvidal@gmail.com, https://orcid.org/0000-0001-6953-6223. ${ }^{3}$ Dr. Facultad de Ciencias Forestales, Universidad de Talca, Talca, Chile, mponce@utalca.cl, https://orcid.org/0000-0001-9949-2057. ${ }^{4}$ M. Sc. Colegio Agrícola de Colín, Talca, Chile, mmedozaalvarez@gmail.com, https://orcid.org/0000-0002-3478-3416.
\end{abstract}

Autor para correspondencia: Oscar VallejosBarra. Dr. Facultad de Ciencias Forestales, Universidad de Talca. Chile. 1 poniente 1141, Talca, Chile, ovallejo@utalca.cl, https://orcid. org/0000-0002-0155-0835.

Citación sugerida / Suggested citation: VALLEJOS-BARRA, O.; VIDAL PAIVA, C.; PONCE-DONOSO, M.; MENDOZA ÁLVAREZ, M. 2019. Detección de Oryctomorphus bimaculatus (Coleoptera: Scarabaeidae: Rutelinae) en Platanus orientalis, Talca, Chile. Revista Colombiana de Entomología 45 (1): e7816 https://doi.org/10.25100/socolen.v45i1.7816

Recibido: 16-nov-2017

Aceptado: 26-nov-2018

Publicado: 31-ago-2019

Revista Colombiana de Entomología ISSN (Impreso): 0120-0488

ISSN (En línea): 2665-4385

http://revistacolombianaentomologia.univalle.edu.co/

Open access

Publicadores / Publishers:

Sociedad Colombiana de Entomología

SOCOLEN (Bogotá, D. C., Colombia)

$\mathrm{http}: / /$ www.socolen.org.co

Universidad del Valle (Cali, Colombia)

http://www.univalle.edu.co/

(C) 2019 Sociedad Colombiana de Entomología - SOCOLEN y Universidad del Valle - Univalle
Resumen: Se registra por primera vez la presencia del escarabajo xilófago Oryctomorphus bimaculatus en un espécimen de Platanus orientalis, cuya edad superaba los 80 años a finales de la primavera de 2015 en la ciudad de Talca, Chile. Se encontraron varios estadios del escarabajo junto a un grupo de hongos saprófitos. Se procedió a una revisión del árbol con elementos tradicionales y con instrumentos no destructivos para evaluar la dimensión del daño. No se encontró la presencia de estos agentes en otros árboles de la cercanía.

Palabras clave: Sanidad arbórea, insecto, xilófago, arbolado urbano, análisis no destructivo.

Abstract: The presence of the xylophage beetle Oryctomorphus bimaculatus is recorded for the first time in a specimen of Platanus orientalis, whose age exceeded 80 years old at end of the spring of 2015 in Talca city, Chile. Several stages of the beetle were found next to a group of saprophytic fungi. The tree was revised with traditional elements and with non-destructive instruments to evaluate the amount of damage. The presence of these agents was not found in other trees in the vicinity.

Keywords: Tree health, insect, xylophage, urban trees, non-destructive analysis.

\section{Introducción}

En Chile el género Oryctomorphus (Coleoptera, Scarabaeidae, Rutelinae), se encuentra representado por las especies Oryctomorphus bimaculatus (Guérin-Méneville, 1830), Oryctomorphus maculicollis (Guérin-Méneville, 1838) y Oryctomorphus laevipennis (Germain, 1855). La primera es la más polífaga, la cual tiene como especies hospederas a Eriobotrya japonica (Thunb.) Lindl. (Rosaceae), Persea americana Mill. (Laureaceae), Pinus radiata (D. Don) (Pinaceae) y las provenientes de la familia Nothofagaceae Nothofagus dombeyi (Mirb.) Blume, Nothofagus obliqua (Mirb.) Oerst. y Nothofagus pumilio (Poepp. \& Endl.) Krasser, tanto en árboles enfermos como muertos (Barriga et al. 1993; Artigas 1994). Por su parte O. maculicollis se ha encontrado en Puya chilensis Mol. (Bromeliaceae) y Salix babylonica Linneo (Salicaceae), ambas en madera muerta (Barriga et al. 1993). En el caso de O. laevipennis tiene como hospedero a $N$. obliqua (Barriga et al. 1993). En Chile no se tienen registros de la presencia de O. bimaculatus en madera viva o muerta de Platanus orientalis L. (Platanaceae) o en otras especies del arbolado urbano.

Platanus orientalis es una especie arbórea exótica ampliamente utilizada en la arborización de las ciudades de Chile, cuyos ejemplares más longevos presentan edades que superan los 80 años (Ponce-Donoso et al. 2009). Junto con su gran envergadura, se le reconocen una serie de servicios ambientales, destacando la regulación del entorno microclimático, retención del material particulado y dis-servicios ambientales como su potencial alergénico y la emisión de compuestos orgánicos volátiles (Dobbs et al. 2011; Mardones et al. 2013; Muñoz et al. 2017).

\section{Materiales y métodos}

Hallazgo. Platanus orientalis es una de las seis especies estudiadas en el proyecto Fondecyt 1150815 "Protocolo de gestión del arbolado urbano: uso de análisis multisensorial para predecir la calidad sanitaria a nivel individual", que se desarrolló 


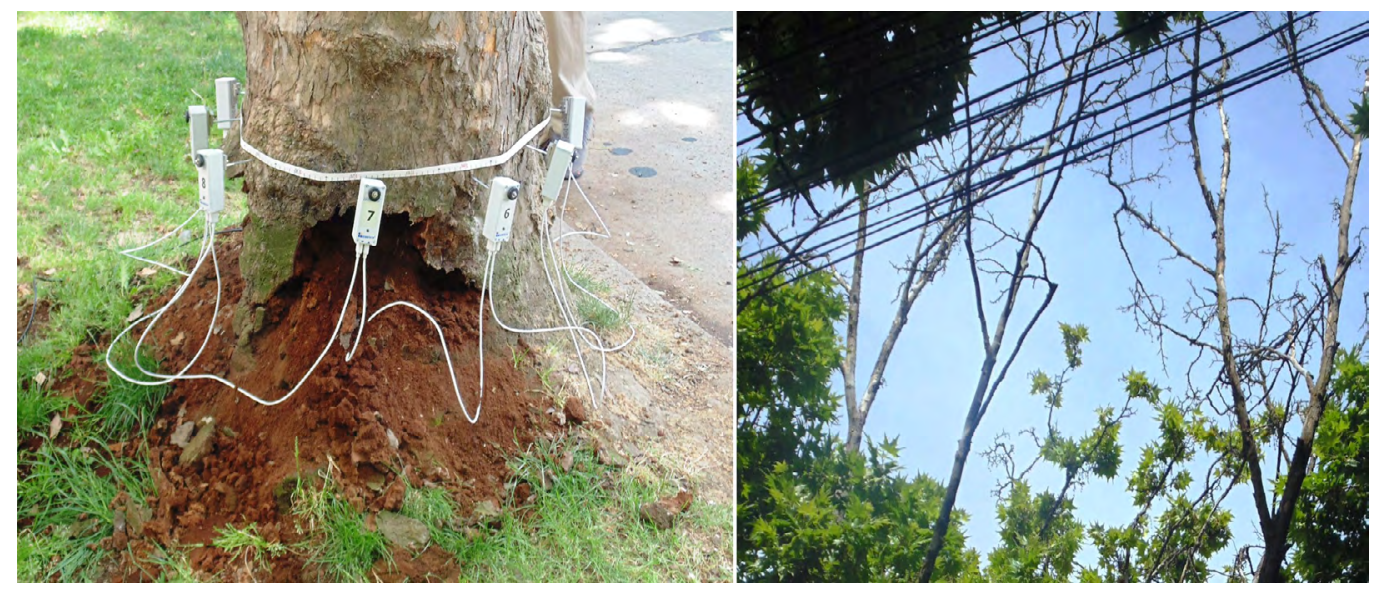

Figura 1. Platanus orientalis dañado por ataque de Oryctomorphus bimaculatus. A. Base del árbol. B. Ramas muertas del árbol.

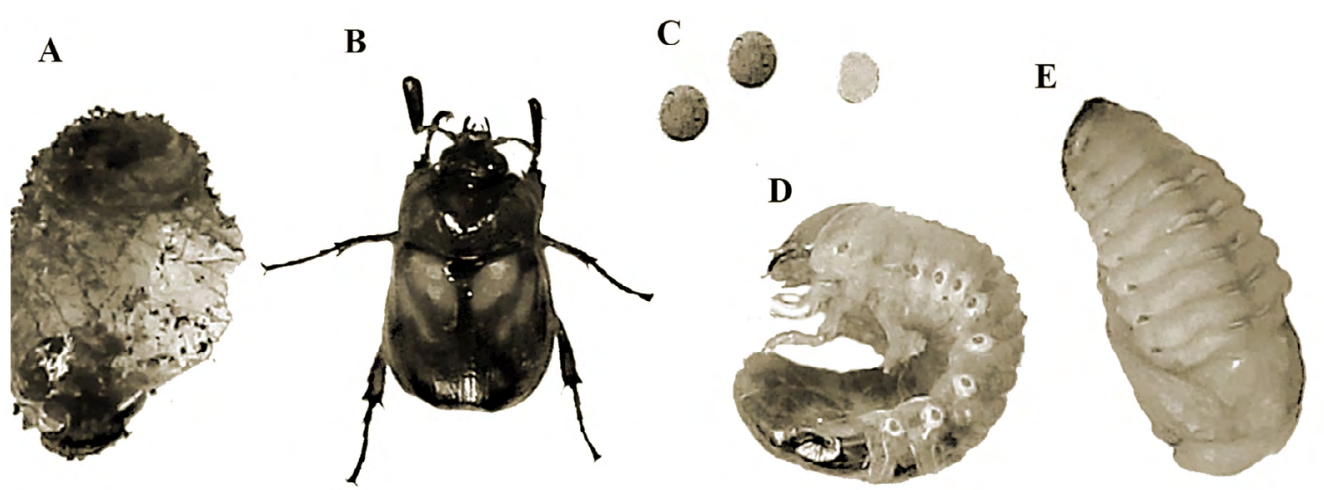

Figura 2. Ejemplares encontrados de Oryctomorphus bimaculatus en distintos estados de desarrollo. A. Exuvia. B. Adulto. C. Huevos. D. Larva. E. Pupa.

en cuatro ciudades de Chile central. Durante la etapa de selección de individuos de esta especie, en calle 3 oriente 6 y 7 norte de la ciudad de Talca $\left(35^{\circ} 25,1^{\prime} \mathrm{S} ; 7^{\circ} 39,7^{\prime} \mathrm{O}\right)$ se identificó un ejemplar que presentaba madera en descomposición y cuerpos fructíferos (Fig. 1), que permitió reportar el hallazgo de $O$. bimaculatus en este espécimen.

Evaluación no destructiva. Se utilizó el tomógrafo sónico Arbotom AT5-E para la evaluación no destructiva de ejemplar; siendo aplicado en dos alturas del árbol. Cuanto mayor sea la presencia de defectos, la velocidad de propagación disminuye, ya que se interrumpe la transmisión de la onda (Secco et al. 2012).

\section{Resultados y discusión}

En un recorrido habitual de medición por la ciudad de Talca el 11 de diciembre de 2015, el equipo investigativo identificó un ejemplar con daño (Fig. 1A), ubicado en calle 3 oriente entre 6 y 7 norte, en cuyas veredas por cerca de 20 calles presentan solo esta especie, que tienen por lo general más de 80 años.

El ejemplar con daño tenía 15 metros de altura y 45 centímetros de diámetro a la altura del pecho. El daño se extendía desde el suelo hasta una altura aproximada de 50 centímetros (Fig. 1A), además presentaba algunas ramas muertas (Fig. 1B), síntoma que podría atribuirse al ataque de algunos agentes xilófagos o bien a un proceso de regresión.
En la base del árbol se observaron orificios de emergencia y aserrín, que abarcaba aproximadamente un $50 \%$ del área basal, revelando la presencia de $O$. bimaculatus en todos los estados de desarrollo (Fig. 2). La literatura espe-

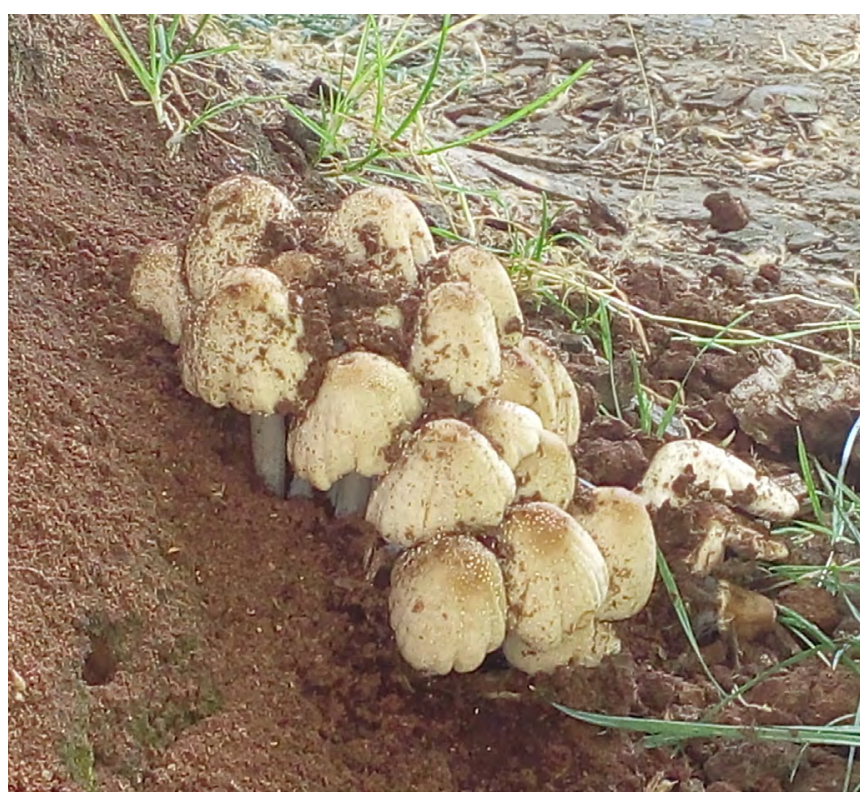

Figura 3. Cuerpos fructíferos de Coprinacae spp. en la base del árbol afectado. 
A

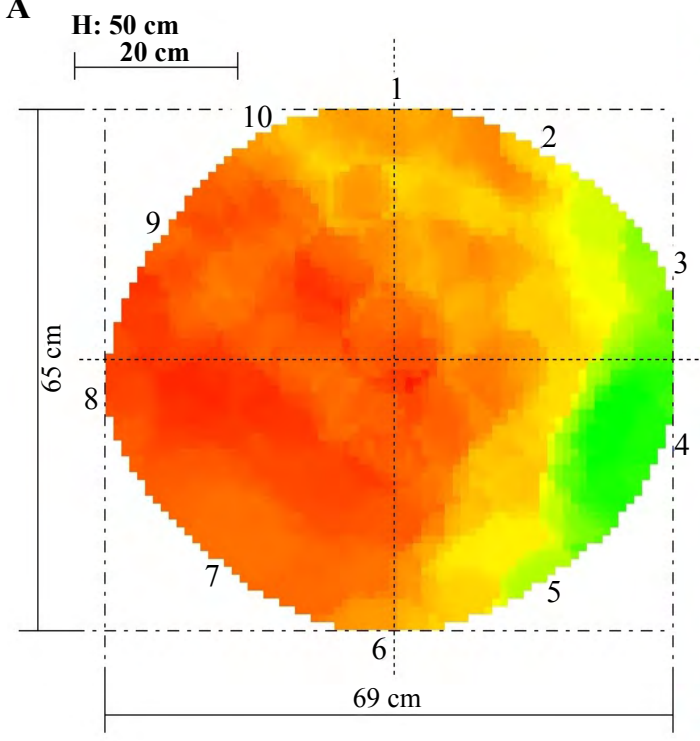

B

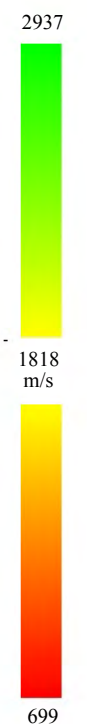

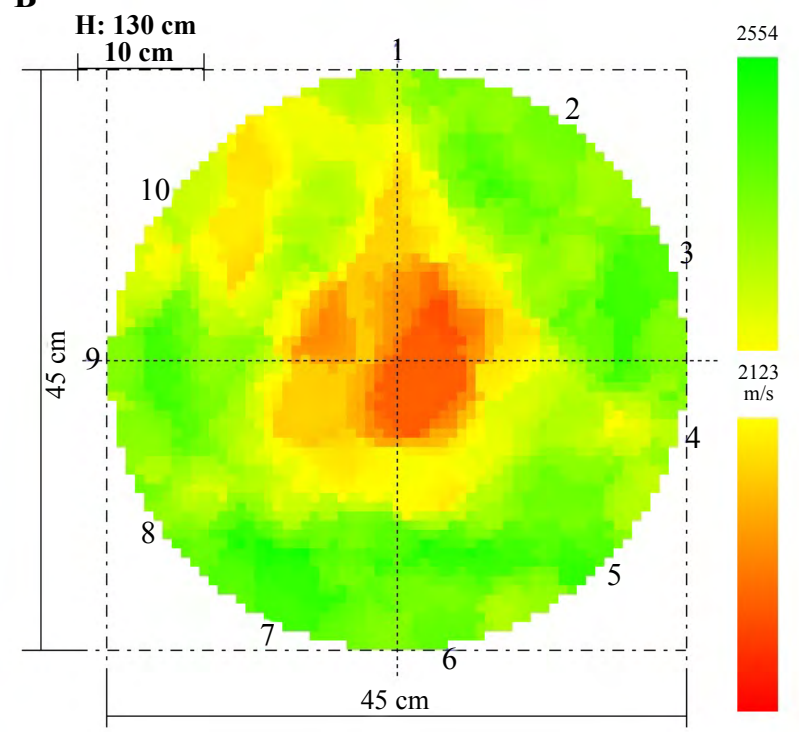

Figura 4. Tomografía del árbol afectado. Las velocidades más altas (color verde) representan una mejor condición de la albura, mientras que las menores velocidades (color rojo) muestra una condición de defecto o descomposición. A. Medición a $50 \mathrm{~cm}$. B. Medición a $1,3 \mathrm{~m}$.

cializada no reporta a P. orientalis como hospedero de este escarabajo.

Junto al hallazgo de los insectos, en la base del tronco se observó un grupo de hongos saprófitos (Fig. 3), de la familia Coprinaceae (Homobasidiomycetes: Agaricomycetidae: Agaricales) comunes en madera muerta (Furci 2007).

No se observaron síntomas o signos de ataque o daño por el insecto en otros ejemplares de $P$. orientalis ubicados en la misma calle, por lo que es de suponer que el evento fue casual, sin manifestar alguna especial preferencia del insecto por este árbol, debido a que este escarabajo se alimenta de madera muerta en proceso de descomposición, siendo una especie oportunista; si no hay más árboles muertos o moribundos, este escarabajo no estaría presente. Un aspecto a destacar de esta situación es notar que $O$. bimaculatus y los escarabeidos en general, se sienten atraídos por luces artificiales (Artigas 1994) de casas y alumbrado público, que podrían ser utilizadas como orientación del vuelo, similar a la que tienen las abejas que utilizan la posición del sol (Márquez 2005), lo que podría contribuir en su llegada en este espécimen arbóreo.

En la aplicación del tomógrafico sónico a los $50 \mathrm{~cm}$ (Fig. 4A) se observó daño superior al $50 \%$ de la sección del tronco y las velocidades fueron similares a las reportadas por Basterrechea Arévalo (2016) para la misma especie (699 a $2.863 \mathrm{~m}$ $\mathrm{s}^{-1}$ ). A los 1,3 m (Fig. 4B), se notó un daño menor, localizado en su centro y la amplitud de las velocidades se restringieron $\left(1.693\right.$ a $\left.2.554 \mathrm{~m} \mathrm{~s}^{-1}\right)$.

Una vez realizado el hallazgo se informó a los encargados de arbolado urbano de la Ilustre Municipalidad de Talca, quienes procedieron a su extracción, a fin de eliminar el riesgo que presentaba el ejemplar.

\section{Agradecimientos}

Los autores agradecen al Fondo Nacional de Desarrollo Científico y Tecnológico de Chile (FONDECYT) por el apoyo recibido para la ejecución del Proyecto 1150815. De igual modo a Jorge Lobos Miranda y Mauro Ríos Venegas, quienes ayudaron en la identificación de la especie y la determinación de su hábito. Agradecemos también a los evaluadores por las observaciones realizadas que permitieron mejorar esta comunicación.

\section{Literatura citada}

ARTIGAS, J. N. 1994. Entomología Económica: insectos de interés agrícola, forestal, médico y veterinario (nativos, introducidos y susceptibles de ser introducidos) Vol. II. Ediciones Universidad de Concepción. Concepción, Chile. 943 p.

BARRIGA T., J. E.; CURKOVIC S., T.; FICHET L., T.; HENRÍQUEZ S., J. L.; MACAYA B., J. 1993. Nuevos antecedentes de coleópteros xilófagos y plantas hospederas en Chile, con una recopilación de citas previas. Revista Chilena de Entomología 20: 65-91.

BASTERRECHEA ARÉVALO, M. A. 2016. Comparación de las técnicas no destructivas de tomografía ultrasónica y resistencia a la perforación en la evaluación de discos de madera. Universidad Politécnica de Madrid, Escuela Técnica Superior de Ingenieros de Montes, Universidade Estadual de Campinas. Proyecto fin de carrera. 1045 p. Disponible en: <http://oa.upm.es/44444/1/PFC MAR_AINHOA_BASTERRECHEA_AREVALO.pdf $>$. [Fechā revisión: 6 marzo 2017].

DOBBS, C.; HERNÁNDEZ, J.; ESCOBEDO F. 2011. Ecuaciones de biomasa aérea y área foliar basadas en métodos no destructivos para árboles urbanos de dos comunas de Chile Central. Bosque 32: 287-296. https://doi.org/10.4067/S071792002011000300010

GEORGE-NASCIMENTO, G. M. F. 2007. Fungi Austral. Guía de campo de los hongos más vistosos de Chile. CORMA. 199 p.

MARDONES, P.; GRAU, M.; ARAYA, J.; CÓRDOVA, A.; PEREIRA, I.; PEÑAILILLO, P.; SILVA, R.; MORAGA, A.; AGUILERA-INSUNZA, R.; YEPES-NÚÑEZ, J. J.; PALOMO, I. 2013. First annual register of allergenic pollen in Talca, Chile. Allergologia et Immunopathologia 41: 233-238. https://doi. org/10.1016/j.aller.2012.06.001

MÁRQUEZ L., J. 2005. Técnicas de colecta y preservación de insectos. Boletín Sociedad Entomológica Aragonesa 37: 385408. 
MUÑOZ, D.; AGUILAR, B.; FUENTEALBA, R.; PRÉNDEZ, M. 2017. Environmental studies in two communes of Santiago of Chile by the analysis of magnetic properties of particulate matter deposited on leaves of roadside trees. Atmospheric Environment 152: 617-627. https://doi.org/10.1016/j.atmosenv.2016.12.047

PONCE-DONOSO, M.; MOYA, L.; BUSTOS-LETELIER, L. 2009. Evaluation of formulas for appraisal of urban trees in municipalities of Chile. Scientia Forestalis 37: 321-329.

SECCO, C.; GONÇALVES, R.; PELEGRINO CERRI, D.; VASQUES, E.; FRANCO BATISTA, F. 2012. Behavior of ultrasonic waves in wood with presence of holes. CERNE 18: 507-514. https://doi.org/10.1590/S0104-77602012000300019

\section{Origen y financiación}

El artículo se basa en el hallazgo producido durante la ejecución del proyecto "Protocolo de gestión del arbolado urbano: uso de análisis multisensorial para predecir la calidad sanitaria a nivel individual" financiado por el Fondo Nacional de Desarrollo Cientifico y Tecnológico de Chile (FONDECYT) $N^{\circ} 1150815$.

\section{Contribución de los autores}

Durante el hallazgo ocurrido en el desarrollo de las actividades de campo, los autores contribuyeron de manera similar. La redacción y corrección de textos y figuras, correspondió a los autores Vallejos-Barra, Vidal Paiva y Ponce-Donoso, que aportaron equitativamente en el trabajo. La revisión técnica fue realizada principalmente por el autor Vidal Paiva y apoyado por el autor Vallejos-Barra. 